\title{
Dose-Exposure Proportionality of a Novel Recombinant Follicle- Stimulating Hormone (rFSH), FE 999049, Derived from a Human Cell Line, with Comparison Between Caucasian and Japanese Women After Subcutaneous Administration
}

\author{
Håkan Olsson • Rikard Sandström • \\ Yu Bagger
}

Published online: 14 March 2015

(c) The Author(s) 2015. This article is published with open access at Springerlink.com

\begin{abstract}
Background and Objectives FE 999049 is a novel recombinant follicle-stimulating hormone $(\mathrm{rFSH})$ preparation expressed by a human cell line (PER.C6 ${ }^{\circledR}$ ), in contrast to existing $\mathrm{rFSH}$ preparations expressed by Chinese hamster ovary $(\mathrm{CHO})$ cell lines. Since the individual dose of $\mathrm{rFSH}$ may be altered depending on the response in women undergoing assisted reproductive technologies, knowledge on the dose-exposure linearity and proportionality is important. The purpose of these studies was to investigate the dose-exposure linearity and proportionality properties of FE 999049 with a comparison between Caucasian and Japanese women. This is the first study in Japanese women regarding pharmacokinetics of $\mathrm{rFSH}$.

Methods Forty-eight Caucasian and 31 Japanese healthy women of reproductive age were pituitary down-regulated to suppress endogenous FSH. Following single subcutaneous administration of $37.5,75,150,225$, or $450 \mathrm{IU}$ (Steelman-Pohley assay), the serum FSH concentration was followed over 10 days.

Results The dose-dependent pharmacokinetic parameters of FE 999049, area under the serum concentration-time curve (AUC) and maximum serum concentration $\left(C_{\max }\right)$, showed dose-exposure linearity and proportionality over $150-450$ IU in Caucasian women, the dose interval available for analysis, and 75-450 IU in Japanese women, which was the dose interval investigated. Comparison between Caucasian and Japanese women showed no differences between the populations. The dose-independent parameters were similar over all doses in both populations.
\end{abstract}

H. Olsson $(\bowtie) \cdot$ R. Sandström · Y. Bagger

Department of Experimental Medicine, Ferring Pharmaceuticals

A/S, Kay Fiskers Plads 11, 2100 Copenhagen, Denmark

e-mail: hakan.olsson@ferring.com
FE 999049 was safe and well tolerated at all doses in both populations with few, mostly mild, adverse events.

Conclusion The results demonstrate dose-exposure proportionality and a predictable dose-dependent exposure of FE 999049, with no differences in Caucasian and Japanese women of reproductive age.

\section{Key Points}

Dose-exposure proportionality for both AUC and $C_{\text {max }}$ was demonstrated for the novel $\mathrm{rFSH}$ FE 999049 in the dose range 75-450 IU in Caucasian and Japanese women.

There were no differences in pharmacokinetic properties and dose proportionality between Caucasian and Japanese women, this being the first report on the pharmacokinetics of $\mathrm{rFSH}$ in a Japanese population.

The results provide important information for the selection of dose when a change in dose is required during the treatment with $\mathrm{rFSH}$ in women undergoing assisted reproductive technologies.

\section{Introduction}

Follicle-stimulating hormone (FSH) is a key hormone in both male and female reproductive functions. It is produced in the anterior pituitary gland and stimulates growth and maturation of ovarian follicles in women [1] while it 
promotes spermatogenesis in men [2]. The synthesis and release of FSH is mainly controlled by the secretion of gonadotropin-releasing hormone (GnRH) from the hypothalamus. In addition to the ovaries, animal data indicate that FSH is distributed to the kidney and liver [3, 4], and mainly eliminated by the kidney $[4,5]$.

FSH is a glycoprotein composed of two non-covalently bound polypeptide chains, denoted $\alpha$ and $\beta$. Pituitary FSH, pituitary luteinizing hormone (LH), pituitary thyroid-stimulating hormone (TSH), and placental chorionic gonadotropin (hCG) all share the same $\alpha$-subunit. In contrast, the structurally different $\beta$-subunit is unique to each of the hormones. Both subunits of FSH are N-glycosylated at two positions, the carbohydrates accounting for nearly onethird of the total mass, which give rise to numerous isoforms of the molecule with substantial variations in size and structure of the carbohydrate chains as well as the level of sialylation $[6,7]$. The composition of the carbohydrate moieties affects the activity of FSH in vivo by influencing the clearance, the binding properties to the FSH receptor, and the ability to activate the receptors. Generally, more acidic isoforms have a lower clearance [8-10], while a higher number of sialic acid residues has commonly been reported to be associated with increased biological activity in vitro and in vivo [8, 11-14].

Since the introduction of recombinant FSH ( $\mathrm{rFSH})$ in the mid-1990s it has been widely used in the treatment of infertility. Currently, the available rFSH products used for controlled ovarian stimulation (COS) in women undergoing in vitro fertilization (IVF) or intracytoplasmic sperm injection (ICSI) cycles, i.e., follitropin $\alpha$ and follitropin $\beta$, are expressed in Chinese hamster ovary (CHO) cell lines [15]. Recently, a novel rFSH (FE 999049) derived from a cell line of human fetal retinal origin (PER.C6 ${ }^{\circledR}$ ) entered clinical development. The amino acid sequences of the $\alpha$ and $\beta$-subunits of FE 999049 are identical to the sequence of natural human FSH and existing $\mathrm{CHO}$-derived $\mathrm{rFSH}$ products in clinical use. However, the sialic acid content of FE 999049 is different and more complex, with both a2,3 and a2,6 sialylation compared with the CHO-derived FSH products, which only contain a2,3 sialylation, and thus more resembling the natural human $\mathrm{FSH}$ than products from CHO cell lines [16].

FE 999049 has previously been reported to have a lower clearance with higher exposure and pharmacodynamic response compared to CHO-derived rFSH [17] when equally dosed by bioactivity as assessed in rats (Steelman-Pohley assay [18]). Dose proportionality which is an important property in order to predict exposure and the consequent ovarian response when increasing or decreasing the dose, was not investigated in this study. In addition, since the need for assisted reproductive technologies is growing worldwide, there needs to be an understanding of the conformity in different populations. This report presents results from the first-in-human study in Caucasian women, and a dedicated rFSH study in Japanese women, investigating the pharmacokinetic dose-exposure proportionality properties of FE 999049, a novel rFSH derived from a human cell line, with comparisons between the two populations.

\section{Methods}

\subsection{Study Design}

The study protocols and informed consent documents were reviewed and approved by Independent Investigational Review Board Inc., Plantation, Florida, USA, and Compass IRB, Mesa, Arizona, USA, respectively. The studies were conducted in accordance with Declaration of Helsinki and the principles of Good Clinical Practice at Clinical Pharmacology of Miami, USA (Caucasian study), and West Coast Clinical Trials, LLC, Cypress, California, USA (Japanese study). Prior to screening, written informed consent was obtained from all subjects.

The investigations were designed as double-blind, placebo-controlled, within-group randomized, parallelgroup studies. There were five ascending dose panels of FE 999049, 37.5, 75, 150, 225, and 450 IU, in Caucasian women ('Caucasian' study) and four ascending dose panels, 75, 150, 225, 450 IU, in Japanese women ('Japanese' study) with eight healthy women in each dose panel (six active treatment, two placebo). A direct comparison between Japanese and Caucasian women was made by adding eight Caucasian women (six active, two placebo) at the highest dose, $450 \mathrm{IU}$, to the 'Japanese' study.

Subjects found to be eligible started a high-dose combined oral contraceptive (OGESTREL 0.5/50, Watson Pharma Inc., Morristown, NJ, USA) run-in period before administration of rFSH to suppress endogenous FSH, and daily administrations of combined oral contraceptive then continued throughout the study. Three days and one day before administration of rFSH, blood samples were collected for analysis and eligibility check of the FSH and estradiol concentrations. If FSH was $>5 \mathrm{IU} / \mathrm{L}$ or estradiol was $>50 \mathrm{pg} / \mathrm{ml}$ in any of the measurements the subject was excluded from the study.

On the day of rFSH administration, each subject was randomized to receive a bolus subcutaneous injection of either FE 999049 or placebo according to a computergenerated randomization list with a block size of 8 , provided by an independent statistician. Blood samples for pharmacokinetic analysis were collected pre-dose, and 4, 8, $12,16,20,24,28,32,36,40,44,48 \mathrm{~h}$, and 3, 4, 5, 6, 7, 8, and 9 days after administration. 


\subsection{Subjects}

Healthy Caucasian and Japanese women, 21-35 years of age, were enrolled. The main inclusion criteria were body mass index (BMI) between 18 and $29 \mathrm{~kg} / \mathrm{m}^{2}$, menstrual cycle of 24-35 days, and with no clinically significant findings in gynaecological examinations, vital signs, ECG, or laboratory parameters.

\subsection{Sources and Analysis of FE 999049}

All references to FSH activity (IU) are based on the method by Steelman and Pohley [18]. FE 999049 was produced in a cell line of human fetal retinal origin $\left(\right.$ PER.C6 ${ }^{\circledR}$ ) and provided as a sterile phosphate-buffered solution of pH 6-7. The concentration of the active ingredient was $600 \mathrm{IU} / \mathrm{mL}$ FSH activity, to be used undiluted.

Analyses of serum FSH concentrations were performed by the Department of Bioanalysis at Ferring Pharmaceuticals $\mathrm{A} / \mathrm{S}$ by means of a validated immunoassay based on electrochemiluminiscence (ECL). Briefly, a 96-well microplate was coated with a mouse anti-human FSH antibody (AbD Serotec, Oxford, UK) over night, after which samples were transferred by the aid of a Tecan Genesis Freedom sample handling robot. After adding the MSDSULFO-TAG labeled mouse anti-human FSH antibody and incubating for $1 \mathrm{~h}$, the color was developed and read with an MSD Sector Imager 2400. The lower limit of quantification for FE 999049 was $1.32 \mathrm{mIU} / \mathrm{mL}$ as determined from QC samples. The bias at all levels was within $\pm 9 \%$, and the bias for back-calculated calibration samples within $8 \%$.

\subsection{Statistical Analyses}

The pharmacokinetic parameters of FE 999049 were calculated for baseline corrected data, assuming a constant background FSH concentration, by non-compartmental analysis (NCA) using the software WinNonlin ${ }^{\circledR}$ Pro (Pharsight Corporation, Cary, NC, USA). The baseline value was the mean of the values obtained prior to the first administration of rFSH. Serum concentration values below the lower limit of quantitation and missing values (e.g., no blood sample collected or no value obtained at analysis) were excluded from the NCA. Actual time-points for blood sampling were used in the calculations of the individual parameters, while nominal time-points are denoted for summary statistics. Pharmacokinetic parameters were estimated based on measurements from Day 1 to the last day of pharmacokinetic assessment, i.e., Day 10.

Descriptive statistics were calculated for serum concentrations and pharmacokinetic parameters, and all pharmacokinetic parameters were compared across treatment groups using an ANOVA model for the log-transformed values. The ratios of the pharmacokinetic parameters were estimated with $90 \%$ confidence limits. All statistical analyses were performed using SAS software version 9.2 for Windows (SAS Institute Inc., Cary, NC, USA).

\section{Results}

\subsection{Study Subjects}

Seventy-nine healthy women, 48 Caucasian and 31 Japanese, aged between 21 and 35 years were randomized and dosed. The bodyweight ranged from 47 to 90 and 43 to $74 \mathrm{~kg}$ for the Caucasians and Japanese women, respectively, and the corresponding BMI ranged from 18.7 to 28.9 and 18.2 to $28.1 \mathrm{~kg} / \mathrm{m}^{2}$, respectively. The treatment groups within each study were comparable with respect to all demographic parameters (Table 1) as well as concomitant medication and medical history. All subjects completed the study.

\subsection{Pharmacokinetic Evaluation}

Following a single subcutaneous administration of FE 999049 the serum FSH concentration increased to reach a maximum approximately $20 \mathrm{~h}$ after injection in all dose groups in both Caucasian and Japanese subjects. The FSH levels subsequently declined slowly with an approximate terminal half-life of $40 \mathrm{~h}$, similar in all dose groups in both populations (Fig. 1). All placebo subjects except one had at all time-points serum FSH levels below limit of quantitation.

The dose-dependent pharmacokinetic parameters of FE 999049, area under the serum concentration-time curve (AUC) and maximum serum concentration $\left(C_{\max }\right)$, increased with increasing doses in both Caucasian (Table 2) and Japanese (Table 3) women. Analysis of the dose proportionality indicated a linear relationship (all correlation coefficients $>0.985$ ) for both AUC and $C_{\max }$, (Fig. 2) with slopes being 1.0, or close to, thus showing dose proportionality (Table 4). Comparison of the results in the Japanese and Caucasian women between trials did not show any significant differences, and none of the dose-dependent parameters showed any significant difference between the two populations. In addition, the head-to-head comparison between Japanese and Caucasian subjects administered 450 IU in the same study showed very similar results on both AUC and $C_{\max }$ for the two groups (Table 2).

The dose-independent pharmacokinetic parameters remained similar over the dose range, both within and 
Table 1 Summary of demographic characteristics according to FE 999049 dose

\begin{tabular}{llllllll}
\hline Characteristic & $37.5 \mathrm{IU}$ & $75 \mathrm{IU}$ & $150 \mathrm{IU}$ & $225 \mathrm{IU}$ & $450 \mathrm{IU}$ & $450 \mathrm{IU} \mathrm{a}^{\mathrm{a}}$ & Placebo \\
\hline Caucasians & $N=6$ & $N=6$ & $N=6$ & $N=6$ & $N=6$ & $N=6$ & $N=12^{\mathrm{b}}$ \\
Age (years) & $30.7(5.2)$ & $30.1(4.5)$ & $26.8(3.0)$ & $30.9(3.7)$ & $28.0(5.1)$ & $26.2(3.3)$ & $28.9(3.1)$ \\
Weight $(\mathrm{kg})$ & $59.4(9.2)$ & $70.8(6.1)$ & $68.4(14.8)$ & $61.5(5.3)$ & $61.6(6.6)$ & $61.9(7.1)$ & $66.3(11.0)$ \\
BMI $\left(\mathrm{kg} / \mathrm{m}^{2}\right)$ & $23.5(2.5)$ & $26.2(2.1)$ & $24.3(4.3)$ & $24.3(2.2)$ & $23.7(3.0)$ & $22.5(2.6)$ & $25.0(3.5)$ \\
Japanese & & $N=6$ & $N=6$ & $N=5$ & $N=6$ & $N=8$ \\
Age (years) & NA & $29.0(4.1)$ & $29.6(4.3)$ & $26.6(3.0)$ & $26.8(4.2)$ & $27.0(4.7)$ \\
Weight $(\mathrm{kg})$ & NA & $52.5(6.3)$ & $49.8(2.9)$ & $60.7(7.0)$ & $54.9(7.8)$ & $55.6(9.0)$ \\
BMI $\left(\mathrm{kg} / \mathrm{m}^{2}\right)$ & NA & $20.1(1.3)$ & $20.3(1.5)$ & $23.4(2.3)$ & $20.8(2.9)$ & $21.2(3.0)$ \\
\hline
\end{tabular}

Values are means with standard deviation

$B M I$ body mass index, $N$ number of subjects, $N A$ not applicable

a Participating in the 'Japanese' study

b Two of whom from the 'Japanese' study
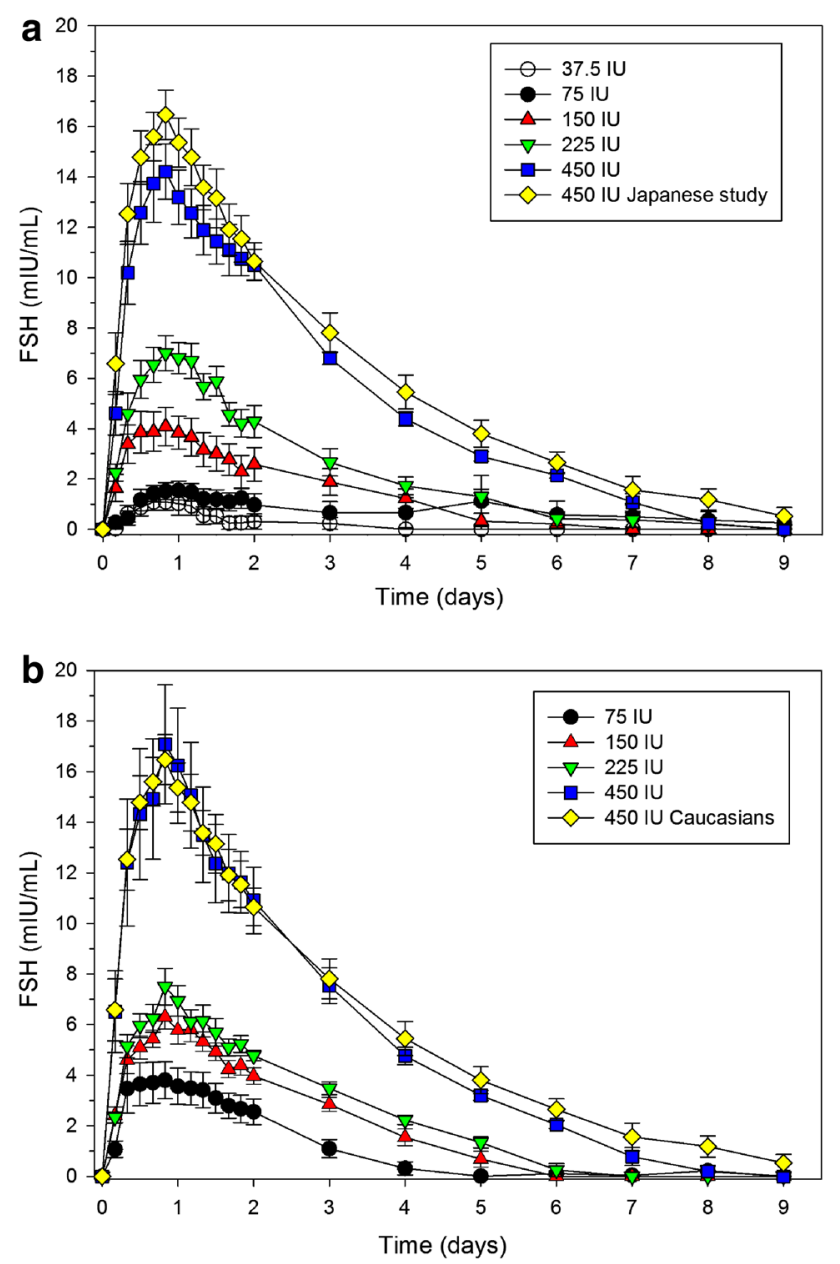

Fig. 1 Time courses of serum FSH mean concentrations after single subcutaneous administrations of FE 999049 to healthy women. a Caucasian women including the group participating in the 'Japanese' study. b Japanese (75-450 IU) and Caucasian (450 IU) healthy women. Values are mean \pm standard error of mean between the two populations without any significant differences between doses or populations (Tables 2, 3).

\subsection{Safety}

All doses were safe and well tolerated in the Caucasian and Japanese subjects. The safety profile of FE 999049 in this trial was acceptable with a few adverse events occurring sporadically in all treatment groups, including the placebo groups, and almost all being of mild intensity. Ten and seven of the adverse events reported by nine ( $25 \%)$ Caucasian and four (17\%) Japanese subjects on active treatment were regarded as related to treatment, and also six $(50 \%)$ and two $(25 \%)$ Caucasian and Japanese placebo subjects reported eight and three adverse events that were regarded as related to treatment. With few exceptions the adverse events (AEs) occurred as single events in various treatment groups, including the placebo groups, with no apparent relation to dose or subject origin. The most frequently reported related AEs in all of the 79 subjects were headache (nine events), injections site reactions at $30 \mathrm{~min}$ or later (five events), and abdominal pain (four events), occurring in both active treatment as well as placebo groups. All related AEs were mild except for one moderate headache, and no subject discontinued due to an AE. The injection site reactions were generally mild and of short duration, occurring also in the placebo groups.

There were no apparent trends or dose-related changes in any of the laboratory parameters clinical chemistry, including liver and kidney functions, haematology, haemostasis, vital signs, or ECG parameters, and no differences were observed between Caucasian and Japanese subjects. No laboratory parameter value was regarded as clinically significant. 
Table 2 Pharmacokinetic parameters of FE 999049 in Caucasian women following a single subcutaneous administration according to FE 999049 dose

\begin{tabular}{|c|c|c|c|c|c|}
\hline \multirow[t]{2}{*}{ Parameter } & \multicolumn{3}{|l|}{ 'Caucasian' study } & \multirow{2}{*}{$\begin{array}{l}\text { 'Japanese' study } \\
450 \mathrm{IU}(N=6)\end{array}$} & \multirow{2}{*}{$\begin{array}{l}\text { Studies combined } \\
450 \mathrm{IU}(N=12)\end{array}$} \\
\hline & $150 \mathrm{IU}(N=6)$ & $225 \mathrm{IU}(N=6)$ & $450 \mathrm{IU}(N=6)$ & & \\
\hline AUC (mIU'h/mL) & 325 (184) & $519(171)$ & $1158(138)$ & $1258(215)$ & $1207(180)$ \\
\hline $\mathrm{AUC}_{t}(\mathrm{mIU} \cdot \mathrm{h} / \mathrm{mL})$ & $254(162)$ & 447 (153) & $1052(136)$ & $1187(227)$ & $1120(191)$ \\
\hline$C_{\max }(\mathrm{mIU} / \mathrm{mL})$ & $4.4(1.9)$ & $7.2(1.7)$ & $14.7(3.2)$ & $16.1(1.9)$ & $15.4(2.6)$ \\
\hline$t_{\max }(\mathrm{h})$ & $20(8-24)$ & $20(16-28)$ & $20(16-24)$ & $20(20-20)$ & $20(16-24)$ \\
\hline$t_{1 / 2}(\mathrm{~h})$ & $39(8)$ & $40(21)$ & $44(6)$ & $43(6)$ & $44(6)$ \\
\hline $\mathrm{CL} / \mathrm{F}(\mathrm{L} / \mathrm{h})$ & $0.70(0.56)$ & $0.50(0.26)$ & $0.39(0.05)$ & $0.37(0.06)$ & $0.38(0.05)$ \\
\hline$V_{\mathrm{z}} / F(\mathrm{~L})$ & $34(19)$ & $24(9)$ & $25(6)$ & $23(3)$ & $24(5)$ \\
\hline
\end{tabular}

Values are presented as mean (SD) except for $t_{\max }$ values, which are presented as medians with range

$N$ number of subjects, $A U C$ area under the serum concentration-time curve, $A U C_{t}$ AUC from time 0 to time $t$, $C_{\max }$ maximum serum concentration, $t_{\max }$ time to reach $C_{\max }, t_{1 / 2}$ elimination half-life, $C L / F$ apparent clearance, $V_{z} / F$ apparent terminal volume of distribution

Table 3 Pharmacokinetic parameters of FE 999049 in Japanese women following a single subcutaneous administration according to FE 999049 dose

\begin{tabular}{lllll}
\hline Parameter & Japanese & & & \\
\cline { 2 - 4 } & $75 \mathrm{IU}(N=6)$ & $150 \mathrm{IU}(N=6)$ & $553(97)$ & $450 \mathrm{IU}(N=6)$ \\
\hline $\mathrm{AUC}(\mathrm{mIU} \cdot \mathrm{h} / \mathrm{mL})$ & $212(91)$ & $455(96)$ & $469(98)$ & $1252(282)$ \\
$\mathrm{AUC}(\mathrm{mIU} \cdot \mathrm{h} / \mathrm{mL})$ & $153(69)$ & $374(92)$ & $7.6(1.6)$ & $1155(308)$ \\
$C_{\max }(\mathrm{mIU} / \mathrm{mL})$ & $3.2(0.8)$ & $6.4(1.2)$ & $20(20-32)$ & $17.3(5.8)$ \\
$t_{\max }(\mathrm{h})$ & $18(8-20)$ & $20(12-24)$ & $40(2)$ & $43(20-24)$ \\
$t_{1 / 2}(\mathrm{~h})$ & $38(13)$ & $37(5)$ & $0.48(0.12)$ & $0.38(0.09)$ \\
$\mathrm{CL} / \mathrm{F}(\mathrm{L} / \mathrm{h})$ & $0.41(0.17)$ & $0.34(0.08)$ & $28(8)$ & $25(13)$ \\
$V_{\mathrm{z}} / F(\mathrm{~L})$ & $23(16)$ & $18(3)$ & & \\
\hline
\end{tabular}

Values are presented as mean (SD) except for $t_{\max }$ values, which are presented as medians with range

$N$ number of subjects, $A U C$ area under the serum concentration-time curve, $A U C_{t}$ AUC from time 0 to time $t$, $C_{\text {max }}$ maximum serum concentration, $t_{\max }$ time to reach $C_{\max }, t_{1 / 2}$ elimination half-life, $C L / F$ apparent clearance, $V_{\mathrm{z}} / F$ apparent terminal volume of distribution

\section{Discussion}

The wide-spread use of $\mathrm{rFSH}$ in assisted reproductive technologies at doses on-demand, imply a trust that the administered dose results in the expected serum concentration and ovarian response. The results of the present dose-exposure proportionality studies demonstrate that rFSH manufactured from a human cell line (PER.C6 ${ }^{\circledR}$ ), with proven different glycosylation than $\mathrm{CHO}$-derived rFSH, show predictable and consistent pharmacokinetic behaviour after single subcutaneous administrations in Caucasian and Japanese women within a dose range covering the clinical use of rFSH. Pharmacokinetic dose proportionality has previously been demonstrated for CHOderived rFSH up to the maximal tested dose 225 IU [19], and in this study dose-exposure proportionality with the human cell line-derived rFSH was demonstrated even further, up to $450 \mathrm{IU}$.

To our knowledge this is the first dedicated investigation with rFSH in Japanese women. As for Caucasian women,
FE 999049 showed dose-exposure proportionality, and the comparison with Caucasian women showed no statistical differences, clearly demonstrating that the pharmacokinetic characteristics of FE 999049 were similar between these populations. Further, no statistical differences were observed neither within the Caucasian nor the Japanese dose groups when comparing dose normalised dose-dependent pharmacokinetic parameters, and neither were any differences observed comparing the dose-independent parameters. The recently demonstrated linear dose-response relationship in pharmacodynamic effect [20] further substantiates the predictable behaviour of the rFSH derived from the human cell-line, and together with the present data show that similar dosing regimens can be employed in populations of different origin.

The administration of single doses of FE 999049 to healthy women demonstrated very similar concentrationtime profiles over the dose range investigated. The serum concentrations of FE 999049 in the two lowest dose groups in Caucasians were very low, preventing meaningful 

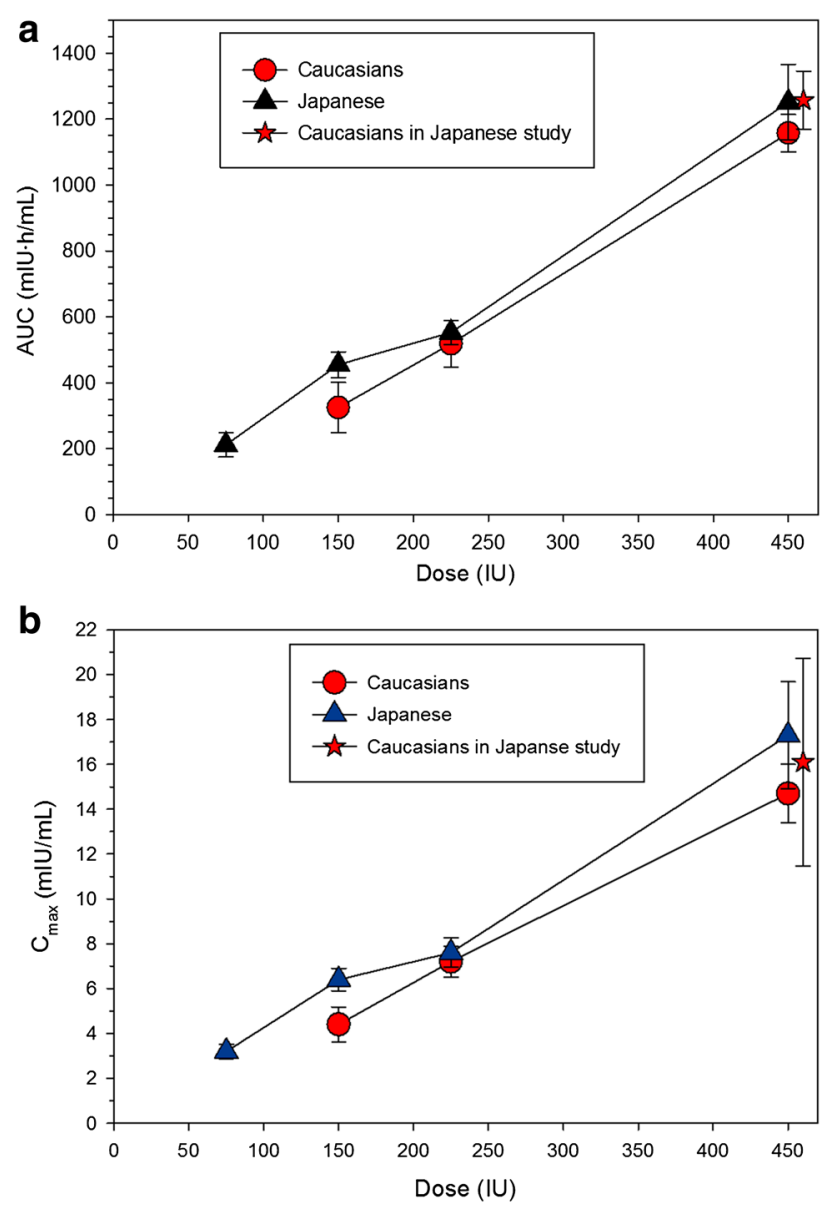

Fig. 2 Dose linearity and proportionality of FE 999049 in healthy women ( $N=6$ per dose). AUC (a) and $C_{\max }$ (b) following single subcutaneous doses of 150-450 IU (Caucasian women) and 75-450 IU (Japanese women). The Caucasian group in the 'Japanese' study is indicated. Values are mean \pm standard error of mean. AUC area under the serum concentration-time curve, $C_{\max }$ maximum serum concentration

pharmacokinetic parameters to be calculated, but the graphical presentation still demonstrated a similar general concentration time-course as for the higher dose groups. Dose-exposure proportionality was demonstrated for AUC and $C_{\max }$ over the range $150-450 \mathrm{IU}$ in Caucasian women and 75-450 IU in Japanese women, thus covering the predominant part of the expected dose range in the clinical setting. The dose-independent kinetic parameters $t_{\max }, t_{1 / 2}$, apparent clearance $(\mathrm{CL} / \mathrm{F})$, and apparent terminal volume of distribution $\left(V_{\mathrm{z}} / F\right)$ were all similar over the dose range and between study populations, supporting the predictability of the pharmacokinetics of varying doses of FE 999049. It is therefore reasonable to assume that doseexposure proportionality extends to $75 \mathrm{IU}$ also for the Caucasian women.

The results for $450 \mathrm{IU}$ of the recombinant human FSH in this study compared well with the results previously reported with a single subcutaneous dose of $450 \mathrm{IU}$ with
Table 4 Dose proportionality of AUC and $C_{\max }$ after a single subcutaneous administration of FE 999049

\begin{tabular}{|c|c|c|}
\hline Parameter & $\begin{array}{l}\text { Caucasians }(N=18) \\
150-450 \mathrm{IU}\end{array}$ & $\begin{array}{l}\text { Japanese }(N=23) \\
75-450 \mathrm{IU}\end{array}$ \\
\hline
\end{tabular}

\begin{tabular}{lllll}
\hline & Slope estimate & $90 \% \mathrm{CI}$ & Slope estimate & $90 \% \mathrm{CI}$ \\
AUC & 1.31 & $0.88-1.74$ & 1.00 & $0.84-1.15$ \\
$C_{\max }$ & 1.16 & $0.84-1.49$ & 0.91 & $0.77-1.05$ \\
\hline
\end{tabular}

The ratio and confidence intervals are estimated using an ANOVA on the natural logarithm of the parameter and including treatment group as fixed effect. Estimates are least square estimates from the model. $\beta$ was estimated using a linear regression model $\mathrm{Ln}\left(\mathrm{AUC} / C_{\max }\right)=$ $\alpha+\beta \times \operatorname{Ln}($ dose $)$

$N$ number of subjects, $A U C$ area under the serum concentration- time curve, $C_{\max }$ maximum serum concentration

respect to all the pharmacokinetic parameters [17], substantiating the consistent exposure with FE 999049. Compared with literature reports of single subcutaneous administrations of $\mathrm{rFSH}$ of $\mathrm{CHO}$ origin, the exposure tended to be greater by means of both AUC and $C_{\max }[19$, $21,22]$, which is in line with the report on direct comparison at a single dose level between the two rFSH preparations [17].

In order to accurately determine the pharmacokinetic properties of exogenously added $\mathrm{rFSH}$, the suppression of endogenous FSH production is essential. For the purpose of minimising the interference by endogenous FSH in the pharmacokinetic analysis, all women were taking a combined oral contraceptive, which was sufficient to suppress the endogenous FSH to levels $<5 \mathrm{mIU} / \mathrm{mL}$ prior to the administration of FE 999049. The efficacy was demonstrated by $>95 \%$ of the women having endogenous FSH levels at baseline that were below the lower limit of quantitation.

The safety profile of FE 999049 was favourable with only a few AEs occurring sporadically in all treatment groups, including the placebo groups, and almost all being of mild intensity with no differences in the safety profile between Caucasian and Japanese women. Overall, the results did not cause any concerns regarding tolerability.

In conclusion, a single subcutaneous administration of the novel rFSH from a human cell line, FE 999049, showed dose proportionality in the range 37.5-450 IU in healthy Caucasian and Japanese women of reproductive age. Moreover, there were no significant differences in the pharmacokinetic characteristics between the two populations.

\section{Conclusion}

In summary, dose-exposure proportionality for both AUC and $C_{\max }$ was demonstrated for the novel subcutaneous 
rFSH FE 999049 in the dose range 75-450 IU in Caucasian and Japanese women. Moreover, there were no differences in pharmacokinetic properties and dose proportionality between Caucasian and Japanese women in this the first investigation on the pharmacokinetics of $\mathrm{rFSH}$ in a Japanese population. The results provide important information for the selection of dose when a change in dose is required during the treatment with $\mathrm{rFSH}$ in women undergoing assisted reproductive technologies.

Acknowledgments The authors would like to acknowledge all investigators and staff at the study centres. The trials were sponsored by Ferring Phamraceuticals.

Conflict of interest Håkan Olsson, Rikard Sandström, and Yu Bagger are employees of Ferring Pharmaceuticals A/S.

Open Access This article is distributed under the terms of the Creative Commons Attribution Noncommercial License which permits any noncommercial use, distribution, and reproduction in any medium, provided the original author(s) and the source are credited.

\section{References}

1. Hillier SG. Current concepts of the roles of follicle stimulating hormone and luteinizing hormone in folliculogenesis. Hum Reprod. 1994;9:188-91.

2. Simoni M, Weinbauer GF, Gromoll J, Nieschlag E. Role of FSH in male gonadal function. Ann Endocrinol (Paris). 1999;60:102-6.

3. Butt WR, Ryle M, Shirley A. Kinetic studies with 125I-labelled follicle-stimulating hormone. J Endocrinol. 1973;58:275-87.

4. Sebok K, Sairam MR, Cantin M, Mohapatra SK. Distribution of follitropin and deglycosylated follitropin in the rat: a quantitative and radioautographic study. Mol Cell Endocrinol. 1987;52:185-97.

5. Emmanouel DS, Stavropolous T, Katz AI. Am J Physiol. Role of the kidney in metabolism of gonadotropins in rats. 1984;247:E786-92.

6. Pierce JG, Parsons TF. Glycoprotein hormones: structure and function. Annu Rev Biochem. 1981;50:465-95.

7. Ulloa-Aguirre A, Timossi C, Damián-Matsumura P, Dias JA. Role of glycosylation in function of follicle-stimulating hormone. Endocrine. 1999;11:205-15.

8. Ulloa-Aguirre A, Timossi C, Barrios-de-Tomasi J, Maldonado A, Nayudu P. Impact of carbohydrate heterogeneity in function of follicle-stimulating hormone: studies derived from in vitro and in vivo models. Biol Reprod. 2003;69:379-89.

9. Goochee CF, Gramer MJ, Anderson DC, Bahr JB, Rasmunsen JR. The oligosaccharides of glycoproteins: bioprocess factors affecting oligosaccharide structure and their effect on glycoprotein properties. Biotechnology. 1991;9:1347-55.
10. D’Antonio M, Borrelli F, Datola A, Bucci R, Mascia M, Polletta P, Piscitelli D, Papoian R. Biological characterization of recombinant human follicle stimulating hormone isoforms. Hum Reprod. 1999;14:1160-7.

11. Zambrano E, Zarinan T, Olivares A, Barrios-de-Tomasi J, UlloaAguirre A. Receptor binding activity and in vitro biological activity of the human FSH charge isoforms as disclosed by heterologous and homologous assay systems: implications for the structure-function relationship of the FSH variants. Endocrine. 1999;10:113-21.

12. Barrios-De-Tomasi J, Timossi C, Merchant H, Quintanar A, Avalos JM, Andersen CY, Ulloa-Aguirre A. Assessment of the in vitro and in vivo biological activities of the human folliclestimulating isohormones. Mol Cell Endcrinol. 2002;186:189-98.

13. Loreti N, Fresno C, Barrera D, Andreone L, Albarran SL, Fernandez EA, Larrea F, Campo S. The glycan structure in recombinant human FSH affects endocrine activity and global gene expression in human granulosa cells. Mol Cell Endocrinol. 2013;366:68-80.

14. de Leeuw R, Mulders J, Voortman G, Rombout F, Damm J, Kloosterboer L. Structure-function relationship of recombinant follicle stimulating hormone (Puregon). Mol Hum Reprod. 1996;2:361-9.

15. Howles C. Genetic engineering of human FSH (Gonal-F). Hum Reprod Update. 1996;2:172-91.

16. World Intellectual Property Organisation (WO 2009/127826 Al). Recombinant FSH including $\alpha$ 2,3 and $\alpha 2,6$ sialylation.

17. Olsson H, Sandström R, Grundemar L. Different pharmacokinetic and pharmacodynamic properties of recombinant follicle-stimulating hormone $(\mathrm{rFSH})$ derived from a human cell line compared with rFSH from a non-human cell line. J Clin Pharmacol. 2014;54:1299-307.

18. Steelman SL, Pohley FM. Assay of the follicle stimulating hormone based on the augmentation with human chorionic gonadotropin. Endocrinology. 1953;53:604-16.

19. Voortman G, Mannaerts BMJL, Huisman JAM. A dose proportionality study of subcutaneously and intramuscularly administered recombinant human follicle stimulating hormone (Follistim/ Puregon) in healthy female volunteers. Fertil Steril. 200;73:1187-93.

20. Arce JC, Nyboe Andersen A, Fernández-Sánchez M, Visnova H, Bosch E, García-Velasco JA, Barri P, de Sutter P, Klein BM, Fauser BC. Ovarian response to recombinant human follicle-stimulating hormone: a randomized, anti-Müllerian hormone-stratified, dose-response trial in women undergoing in vitro fertilization/intracytoplasmic sperm injection. Fertil Steril. 2014; 102:1633-40.

21. le Cotonnec J-Y, Porchet HC, Beltrami V, Kahn A, Toon S, Rowland M. Clinical pharmacology of recombinant human follicle-stimulating hormone: part II. Single dose and steady state pharmacokinetics. Fertil Steril. 1994;61:679-86.

22. le Cotonnec J-Y, Loumaye E, Porchet HC, Beltrami V, Munafo A. Pharmacokinetic and pharmacodynamic interactions between recombinant human luteinizing hormone and recombinant human follicle-stimulating hormone. Fertil Steril. 1998;69:201-9. 